\title{
Optimizing the magnetoinductive lens: Improvement, limits, and possible applications
}

Cite as: J. Appl. Phys. 103, 013115 (2008); https://doi.org/10.1063/1.2828176

Submitted: 26 September 2007 . Accepted: 02 November 2007 . Published Online: 15 January 2008

Manuel J. Freire, and Ricardo Marques

\section{ARTICLES YOU MAY BE INTERESTED IN}

Planar magnetoinductive lens for three-dimensional subwavelength imaging Applied Physics Letters 86, 182505 (2005); https://doi.org/10.1063/1.1922074

Experimental demonstration of a $\mu=-1$ metamaterial lens for magnetic resonance imaging Applied Physics Letters 93, 231108 (2008); https://doi.org/10.1063/1.3043725

Experiments on wireless power transfer with metamaterials

Applied Physics Letters 98, 254101 (2011); https://doi.org/10.1063/1.3601927

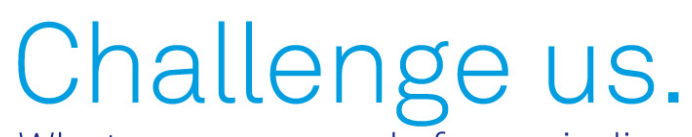

What are your needs for periodic signal detection?

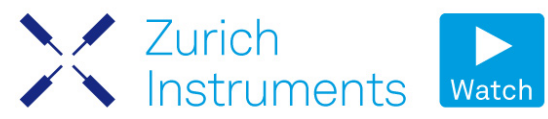

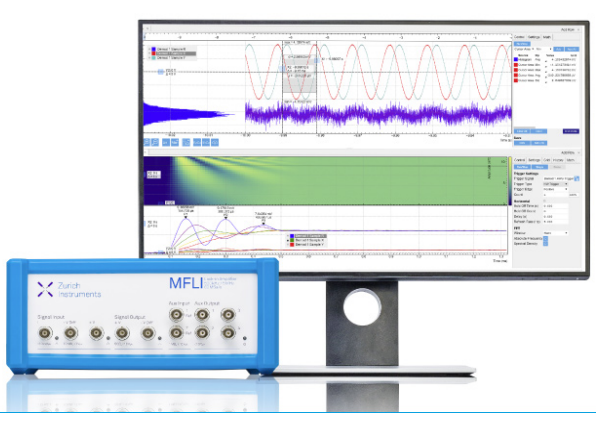

103, 013115 


\title{
Optimizing the magnetoinductive lens: Improvement, limits, and possible applications
}

\author{
Manuel J. Freire ${ }^{\text {a) }}$ and Ricardo Marques \\ Departamento de Electrónica y Electromagnetismo, Facultad de Fisica, Universidad de Sevilla, \\ Avda. Reina Mercedes s/n, E 41012 Sevilla, Spain
}

(Received 26 September 2007; accepted 2 November 2007; published online 15 January 2008)

In this contribution, the basic concepts underlying the physics of magnetoinductive (MI) lenses are developed and the main practical limitations to their performances are analyzed. Strategies to overcome such limitations are proposed. Possible applications of MI lenses in magnetic resonance imaging are discussed. (C) 2008 American Institute of Physics. [DOI: 10.1063/1.2828176]

\section{INTRODUCTION}

The study of metamaterial superlenses overcoming the classical diffraction limit for resolution was initiated by Pendry ${ }^{1}$ in his seminal paper on subwavelength imaging by negative refractive index slabs. An ideal metamaterial perfect lens has to be able to restore, at the image plane, the phase and amplitude of all the Fourier components of the field at the source plane. This includes both propagative and evanescent Fourier harmonics. Therefore, a perfect lens has to be able to amplify evanescent harmonics just by the factor needed to compensate its exponential decay in air. Since the information about the subwavelength details of the source field is carried out by these evanescent components, its amplification inside the lens is the key mechanism for subwavelength imaging. In practice, this Fourier harmonic amplification is limited by material losses and there is a limit for the resolution. It can be shown that the minimum resolution $\Delta$ attainable by a left-handed slab of width $d$ is given by ${ }^{2}$

$$
\Delta \geq \frac{2 \pi d}{\ln (2 / \delta)},
$$

where $\delta$ is the loss tangent of the metamaterial. It is worth noting that, due to the logarithmic dependence on the loss tangent, Eq. (1) implies that the minimum achievable resolution is always of the same order as the slab width for any realistic metamaterial. Therefore, if $\Delta$ should be smaller than the wavelength, $\lambda$, the slab width, $d$, also should be smaller than $\lambda$. That is, superlenses with subdiffraction resolution are near-field devices. This fact suggest the feasibility of designing superlenses operating in the quasielectrostatic and/or the quasimagnetostatic limit. ${ }^{3}$ In Pendry's paper, ${ }^{1}$ it was also shown that a slab with only negative permittivity will produce super-resolution in the quasielectrostatic limit, and it was suggested that a thin silver slab could fulfill this condition in the optical frequency range. This prediction was confirmed experimentally by Fang. ${ }^{4}$ Moreover, Pendry ${ }^{1}$ also pointed out the key role played by surface plasmons for the amplification of evanescent harmonics in the silver slab. This role suggests that other surface waves can also provide super-resolution by the same mechanism in other devices.

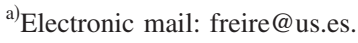

Actually, subdiffraction imaging in the quasimagnetostatic limit was demonstrated for magnetized ferrite slabs operating in the magnetostatic surface wave frequency range. ${ }^{5}$ The fact that the amplification of evanescent harmonics is related with the presence of surface waves rather than with the existence of a negative refractive index was made clear by Maslovski ${ }^{6}$ et al. The structure analyzed in Maslovski's paper ${ }^{6}$ was not a bulky metamaterial slab, but an alternative device consisting of two parallel arrays of resonators in free space, able to support surface waves. In that work ${ }^{6}$ it was also suggested that any system of two coupled plane arrays of resonators supporting surface waves can provide the mechanism for the amplification of evanescent harmonics. If, in addition, such surface waves have a ${ } l a t^{6}$ dispersion relation, then subdiffraction imaging would be possible. Amplification of evanescent harmonics in a specific system made with electric resonators was also verified by Maslovski ${ }^{6}$ by means of an experiment. Subdiffraction imaging in a system of two coupled arrays of magnetic split-ring resonators (SRRs) was experimentally shown ${ }^{7}$ by the authors of the present work, and the complementary superlens, working in the quasielectrostatic limit, was also demonstrated by Ozbay. ${ }^{8}$ Surface waves supported by the SRR arrays used by the authors ${ }^{7}$ are magnetoinductive (MI) surface waves (SWs). Such waves can be excited in any periodic array of inductively coupled magnetic resonators. ${ }^{9}$ For this reason, the reported device consisting of SRR arrays was named MI lens by the authors. ${ }^{7}$ Image formation in MI lenses operating in the radiofrequency range $(\mathrm{MHz})$ was also analyzed by the authors, ${ }^{10}$ both theoretically and experimentally. The theoretical analysis $^{10}$ showed that magnetoinductive surface waves (MISWs) play a role in MI lenses very similar to the role played by surface plasmons in silver lenses. One of the key conclusions of such an analysis was that, for a correct image formation, the MI lens has to be thin enough to avoid the overlapping of the two MISW branches supported by the whole device (these two MISW branches comes from the splitting of the MISWs supported by each isolated array due to the mutual coupling). A similar conclusion was reported by Sydoruk ${ }^{11}$ for a bilayered magnetoinductive linear chain of resonators, following a completely different approach. As for bulky metamaterial lenses, the optimal configuration regarding resolution for MI lenses consists of locating the 


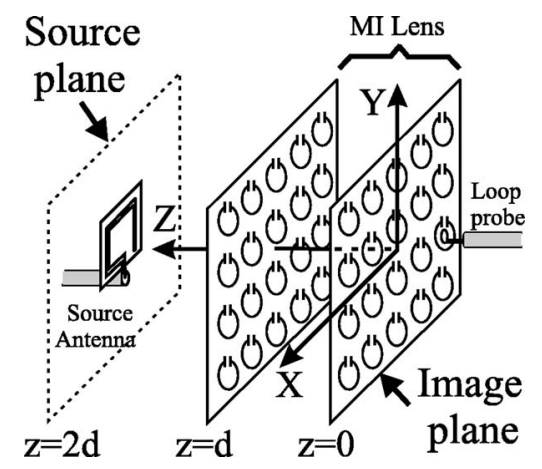

FIG. 1. Schematic representation of the experimental setup used in the study of image formation in the MI lens. A source antenna "illuminates" the MI lens and a loop probe measures the image field at the exit interface of the lens. The MI lens width is $d$. With the exit interface of the lens located at the plane $z=0$, the source plane containing the illuminating antenna is located at a distance twice the MI lens width, that is, at $z=2 d$.

source plane at a distance from the MI lens equal to the MI lens width. ${ }^{10,12}$ In this configuration the image is obtained at the exit interface of the lens ${ }^{4,10,12}$ (see Fig. 1). Thus, the MI lens width determines the imaging distance. On the other hand, the lens resolution $\Delta$ obviously cannot be smaller than twice the MI lens periodicity $a$ (here, we define $\Delta$ as the minimum distance between two pointlike sources that can be resolved by the lens). Therefore, the maximum ratio $d / a$ that can be attained without destroying the super-resolution (i.e., with $\Delta$ still of the same order as $2 a$ ) is the key figure of merit for MI lenses. In the present contribution we will study the theoretical limits for the improvement of such a ratio. First, the role played by resonator losses and inter-resonator coupling will be analyzed qualitatively and quantitatively. Starting from this analysis, we will next discuss some ideas in order to improve MI lens performance, and will check these ideas by experiments carried out in the megahertz range. Finally, some possible applications of MI lenses in magnetic resonance imaging will be discussed.

\section{ANALYSIS}

Figure 1 shows a schematic representation of a MI lens. It consists of two parallel planar periodic arrays of inductively coupled magnetic resonators which can be designed to work at frequencies ranging from megahertz to terahertz $(\mathrm{MHz}$ to $\mathrm{THz}$ ). For devices operating in the $\mathrm{MHz}$ range, these resonators can be metallic rings loaded with a lumped capacitor (with a resonant frequency given by $\omega_{0}=1 / \sqrt{L C}$, where $C$ is the capacitance of the capacitor and $L$ is the ring inductance). For devices operating at higher frequencies, it could be necessary to substitute the lumped capacitor by a distributed capacitance. ${ }^{7}$ Before starting the analysis of the MI lens, we will develop a brief analysis of the behavior of each isolated resonator array. It is well known that this structure supports MISWs with a dispersion relation which, in the nearest-neighbors approximation, is given by ${ }^{9}$

$$
\frac{\omega_{0}^{2}}{\omega^{2}}-\frac{R}{j \omega L}=1+\frac{2 M}{L}\left[\cos \left(k_{x} a\right)+\cos \left(k_{y} a\right)\right],
$$

where $k_{x}, k_{y}$ are the components of the wavevector along the main axis of the array, $a$ is the array periodicity, $M$ is the mutual inductance between the nearest rings, and $R$ is the resistance of the rings. As long as the periodicity $a$ can be considered smaller than the free-space wavelength at the frequency of operation $\lambda$, a continuous approach can be considered for currents and fields. In this approach, an average surface current density $\mathbf{J}_{\mathbf{s}}$ is defined on the array. This surface current density can be related to the average electric field on the surface of the array $\mathbf{E}$ through the array impedance $Z_{a}$,

$$
Z_{a} \mathbf{J}_{s}=\mathbf{E} .
$$

The explicit expression for $Z_{a}$ is ${ }^{10}$

$$
Z_{a}=\frac{j \omega \mu_{0} a^{2}}{k_{x}^{2}+k_{y}^{2}}\left(\frac{1}{\mu_{0} \alpha}-\beta\right)-\frac{1}{2} Z_{0},
$$

where $\alpha$ is the ring magnetic polarizability

$$
\alpha=\frac{\pi^{2} r^{4}}{L}\left(\frac{\omega^{2}}{\omega_{0}^{2}-\omega^{2}+j \omega R / L}\right),
$$

$\beta$ is a coupling factor

$$
\beta=\frac{M}{2 \mu_{0} \pi^{2} r^{4}}\left[\cos \left(k_{x} a\right)+\cos \left(k_{y} a\right)\right],
$$

and $Z_{0}$ is the impedance of transverse electric (TE) waves in free space,

$$
\begin{aligned}
& Z_{0}=\frac{\omega \mu_{0}}{k_{z}}, \\
& k_{z}=\sqrt{\omega^{2} \varepsilon_{0} \mu_{0}-k_{x}^{2}-k_{y}^{2}} .
\end{aligned}
$$

Let us now consider a TE- (or $s$-) polarized wave impinging on the array with a longitudinal wavevector $k_{z}$ $=\sqrt{\omega^{2} \varepsilon_{0} \mu_{0}-k_{x}^{2}-k_{y}^{2}}$. Using Eq. (3), the transmission coefficient through the array $t\left(\omega, k_{x}, k_{y}\right)$ can now be calculated following the general procedure already reported by Maslovski. ${ }^{6}$ The final result is

$$
t=\frac{1}{1+Z_{0} / 2 Z_{a}} \text {. }
$$

It can be easily realized that the roots of the dispersion relation Eq. (2) of magnetoinductive waves correspond to the poles of this transmission coefficient, ${ }^{13}$ i.e., to the solutions of the equation $1 / t=0$. An example of this calculation is given in Fig. 2, where the crest in the plot of $\mid t\left(\omega, k_{x}, k_{y}\right.$ =0) $\mid$ corresponds to the dispersion relation of the MISW Eq. (2). Let us now return to the MI lens shown in Fig. 1. The lens performance is summarized by the transfer function of the lens $T\left(\omega, k_{x}, k_{y}\right)$, which is the transmission coefficient between the source plane $(z=2 d)$ and the image plane $(z$ $=0)$ of Fig. 1. For a correct image formation this transfer function must be a constant for any value of $k_{x}$ and $k_{y}$. The transfer function can be calculated by using the standard transverse transmission matrix technique, ${ }^{6,10,14}$ provided the surface impedance Eq. (3) of each array is known. As an example, a plot of the theoretical values for the modulus of the transfer function $T\left(\omega, k_{x}, k_{y}=0\right)$ for the specific ratio $d / a=0.25$ and the same array parameters as in Fig. 2 is shown in Fig. 3(a). As it can be seen, an almost constant 


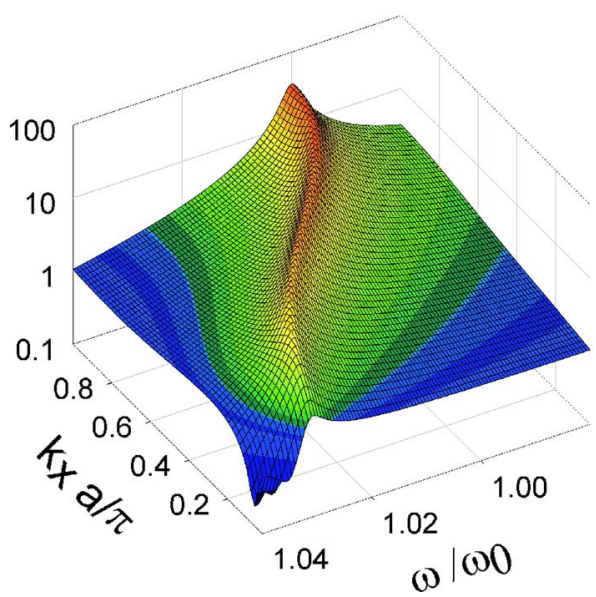

FIG. 2. (Color online) Three-dimensional view of the modulus of the transmission coefficient $t\left(\omega, k_{x}, k_{y}=0\right)$ of a single array of ring resonators as those shown in Fig. 1. The transmission coefficient is plotted as a function of the normalized frequency $\omega / \omega_{0}$ and the normalized wavevector $k_{x} a / \pi$ in the first Brillouin zone, for $k_{y}=0$. Other array parameters are $M / L=-0.015$ and $R / \omega_{0} L=0.003$.

value $\left|T\left(\omega, k_{x}, k_{y}=0\right)\right| \simeq 1$ [in fact $\left.T\left(\omega, k_{x}, k_{y}=0\right) \simeq-1\right]$ appears just in the region between the two peaks of this function, with an optimal frequency cut near the frequency of resonance of the isolated rings $\omega_{0}$. According to our previous analysis, these two peaks of $T\left(\omega, k_{x}, k_{y}\right)$ correspond to the two MISW branches in which the single MISW shown in Fig. 2 splits as a consequence of the coupling between the two arrays. Figure 3(b) shows the value of the modulus and the phase of the transfer function for the best frequency cut (i.e., for the best frequency of operation of the lens $\omega / \omega_{0}$ $=1.018$ ). For most of the values of $k_{x}$, the modulus is equal to 1 and the phase equals $180^{\circ}$ [since $\left.T\left(\omega, k_{x}, k_{y}=0\right) \simeq-1\right]$. As could be expected, when the $d / a$ ratio is increased, this coupling will decrease, and the "valley" of constant $T\left(\omega, k_{x}, k_{y}\right)$ between the two MISW branches will eventually disappear. This result is illustrated in Figs. 3(c) and 3(d), where the theoretical values of the transfer function for $d / a=1$ are shown [the remaining parameters are as in Figs. 3(a) and 3(b)]. Since this reduced coupling for higher $d / a$ ratios is unavoidable, the only way to restore the lens performance would be to reduce the bandwidth of the two MISW branches. According to Eq. (2), this could be achieved by reducing the $M / L$ and/or the $R / \omega_{0} L$ ratios. Since ohmic losses in metallic rings are already very small at radiofrequencies and microwaves, the most promising strategy seems to be to reduce the $M / L$ ratio. Figures 3(e) and 3(f) show the theoretical values of the transfer function $T\left(\omega, k_{x}, k_{y}=0\right)$ for a smaller $M / L$ ratio. As can be seen, the lens performance is restored for this reduced $M / L$ ratio, in agreement with our previous predictions. If we want to further increase the $d / a$ ratio of the lens, we need even smaller values of both $M / L$ and $R / \omega_{0} L$, as shown in Figs. 3(g)-3(j). Therefore, it can be deduced from our analysis that, in order to improve the MI lens performance, reduction of the $M / L$ ratio is a good strategy, and that additional improvements can be achieved by also reducing the $R / \omega_{0} L$ ratio. In any case the attainable values of $\lambda / \Delta \sim 100$ are always of the order of unity. That is, the resolution of MI lenses is always of the

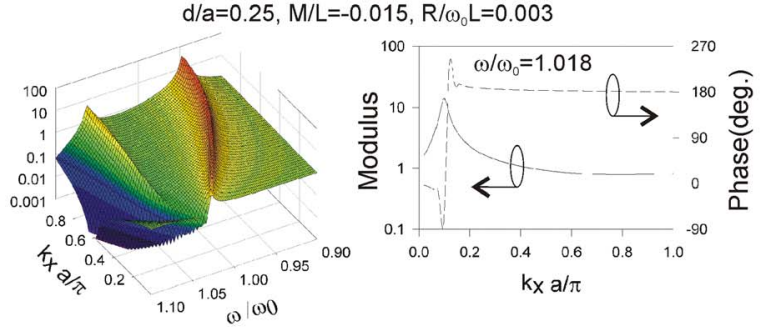

(a)

(b)

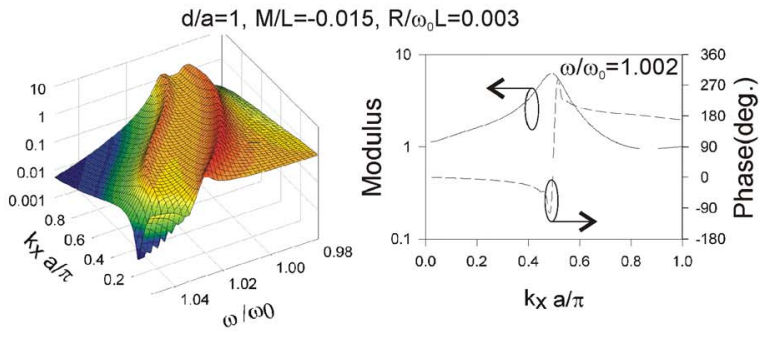

(c)

(d)

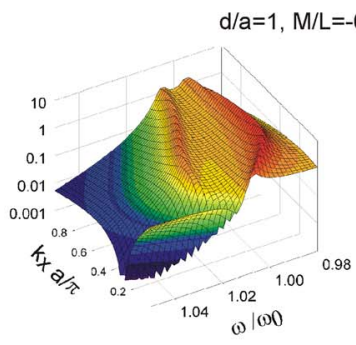

(e)

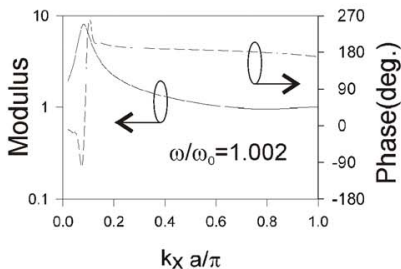

(f)

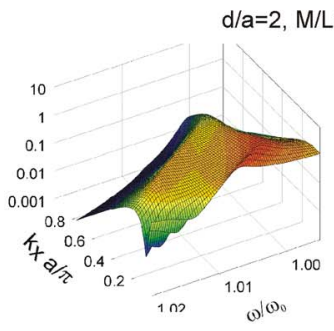

(g)

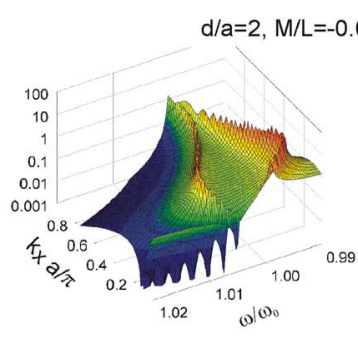

(i)

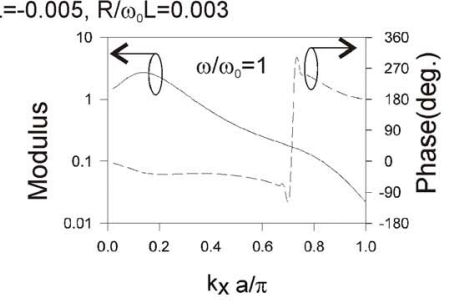

(h)

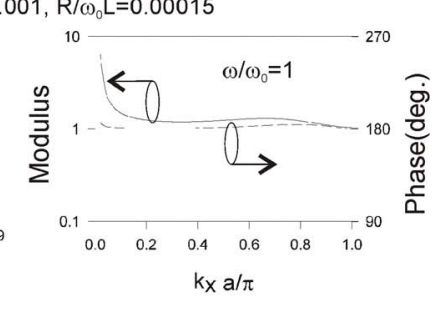

(j)

FIG. 3. (Color online) Three-dimensional view of the modulus of the transfer function of a MI lens as a function of the normalized wavenumber $k_{x} a / \pi$ and the frequency $\omega$ normalized to the resonant frequency $\omega_{0}$, and plot of both the modulus (solid line) and the phase in degree (dashed line) of the transfer function for the best frequency cut.

same order as the lens width for any realistic constitutive materials and geometries. As already noted in the Introduction [see Eq. (1)], the same happens in any other metamate- 
rial superlens. Therefore, the MI lens does not differ in this aspect from other metamaterial imaging devices. The values for the $M / L$ and the $R / \omega_{0} L$ ratios used in our theoretical analysis [Figs. 3(a) and 3(b)] correspond to the usual values for realistic metallic rings (actually they correspond to experimental values ${ }^{10}$ ). Since the self-inductance of a ring $L$ cannot be increased too much (actually it can be increased by substituting the rings by coils, but this will also increase the mutual inductance $M$ ), the only available strategy to reduce the $M / L$ ratio seems to be to reduce $M$. This can be achieved by partially overlapping the rings, so that the "positive" and the "negative" mutual magnetic flux cancel. In fact, this strategy is known in magnetic resonance imaging (MRI) ${ }^{15}$ where it is used in parallel imaging devices in order to decouple the receiving coils. ${ }^{16}$ Regarding the $R / \omega_{0} L$ ratio, it can be reduced by using superconducting rings. At radiofrequencies, superconducting materials have been proven to be useful even in the presence of the high magnetic fields used in MRI. ${ }^{17,18}$ At microwave frequencies, superconducting materials are also available. ${ }^{19}$ Therefore, the aforementioned strategies for the improvement of MI lenses seem to be directly applicable in the frame of present technologies.

\section{EXPERIMENTS}

In order to show by an experiment the feasibility of improving MI lenses by reducing the inter-resonator coupling, a new MI lens has been designed, fabricated, and measured. The resonators of such a lens are circular loops with four prolongations. In the fabrication process, the resonators were photoetched on both sides of a thin microwave circuit board of thickness 127 microns, so that the nearest neighbors overlap, as shown in the photograph in Fig. 4(a). The period of the lens is $a=15 \mathrm{~mm}$, the outer diameter of the circular section is $12 \mathrm{~mm}$, and the strip width is $1 \mathrm{~mm}$. The prolongations were designed with the help of the electromagnetic simulation software ADS/MOMENTUM from Agilent Technologies in order to minimize the $M / L$ ratio between the nearest neighbors. The resonators were loaded with a chip capacitor to provide the necessary capacitance for the resonance. The chip capacitors were provided by Murata and have a nominal capacitance of $82 \mathrm{pF}$, and a tolerance of $5 \%$. With this tolerance, in practice there was a distribution of frequencies significantly greater than the width of any individual resonance. Therefore, the capacitors used for the fabrication of the lenses were carefully measured and selected, so that the tolerance of the capacitors used in the experiment was reduced to $1 \%$. For comparison purposes another MI lens made with simple circular loops was also fabricated and measured [see the photograph in Fig. 4(b)]. In the following, we will call uncoupled-loops MI the (UL-MI) lens for the first lens, and circular-loops MI the (CL-MI) lens for the second lens. For the CL-MI lens, the array parameters are similar to those in Figs. 3(a), that is, $M / L=-0.015$ and $R / \omega_{0} L=0.003(d / a$ for the UL-MI lens). The dimensions of the loops as well as the capacitances and tolerances of the capacitors were the same for both the UL-MI lens and the CL-MI lens. However, a variation in the frequency of resonance can be expected due to the effect of the prolongations on the self-inductance of

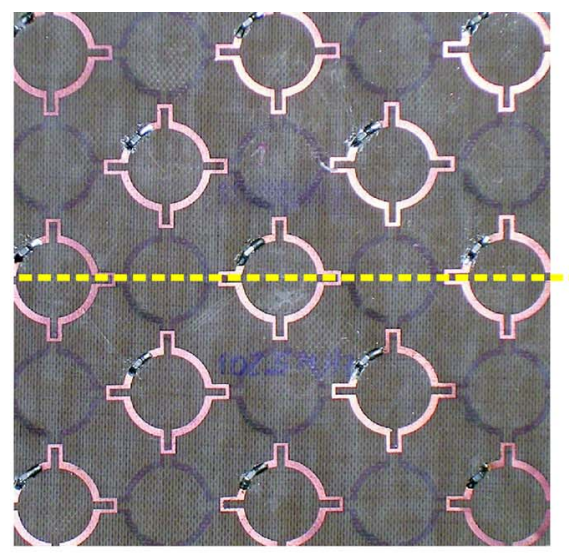

(a)

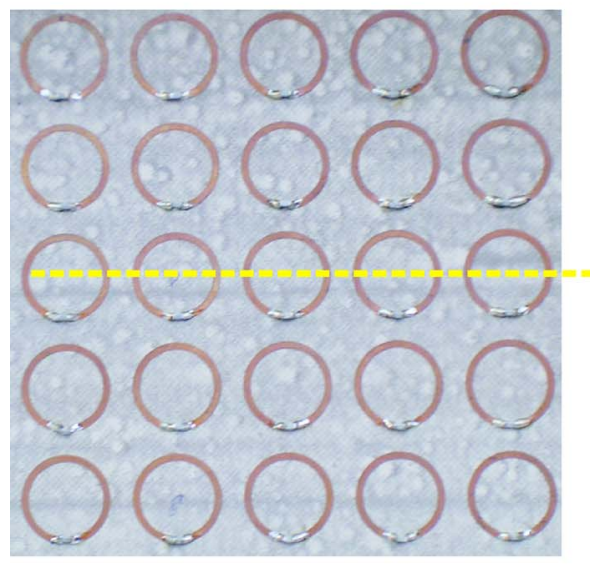

(b)
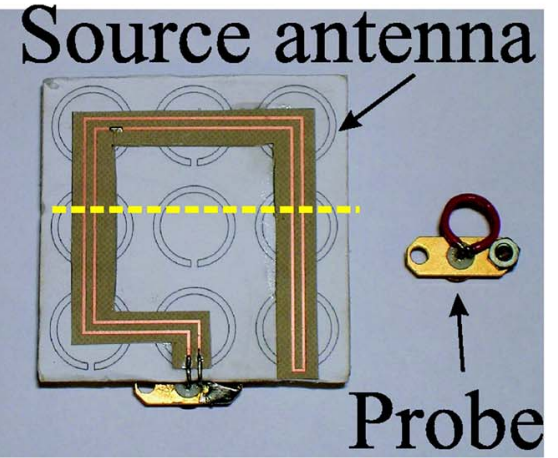

(c)

FIG. 4. (Color online) (a) Photograph of an array of the fabricated UL-MI lens. (b) Photograph of an array of the CL-MI lens. (c) Photograph of the antenna used to provide the magnetic field at the source plane and of the probe used to measure the field. The line along which the field was measured is also shown (yellow dashed line).

the loops in the UL-MI lens. Thus, the resonant frequency $\omega_{0} / 2 \pi$ was $103 \mathrm{MHz}$ for the UL-MI lens and $119 \mathrm{MHz}$ for the CL-MI lens. In the experiments, a planar antenna was placed in the source plane at a distance $d$ from the lens and $2 d$ from the image plane, as shown in the scheme of Fig. 1. This antenna was similar to that used in a previous work of the authors. ${ }^{10}$ It was made of sections of a two-parallel-strip transmission line which was short-circuited at its end. This transmission line section was bent in order to form a square 


\section{Uncoupled loops, freq. $=103.2 \mathrm{MHz}$}

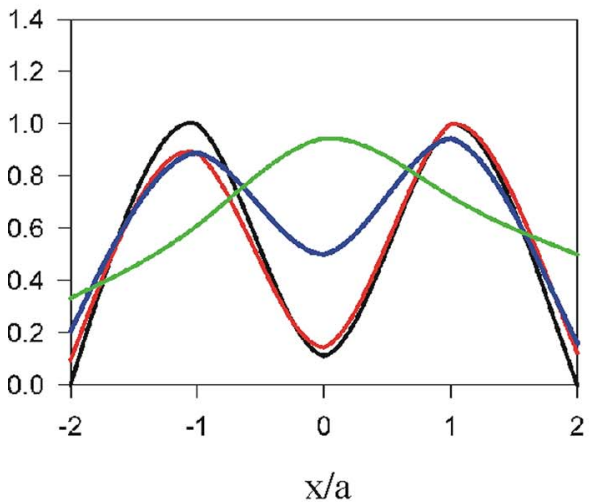

(a)

\section{Circular loops, freq. $=120 \mathrm{MHz}$}

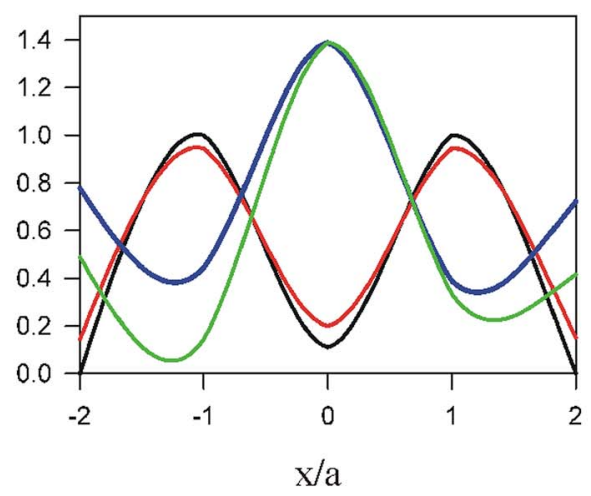

(b)

FIG. 5. (Color online) Source field and image field measured with a probe in both the UL-MI lens at a frequency of $103.2 \mathrm{MHz}$ (a) and in the CL-MI lens at a frequency of $120 \mathrm{MHz}$ (b), for several $d / a$ values. To measure the image field the probe was moved by steps equal to the lens periodicity $a$. The source field corresponds to the black line. The different $d / a$ values are the following: $d / a=0.025$, red line; $d / a=0.45$, blue line; $d / a=0.6$, green line.

loop as shown in Fig. 4(c). The resulting antenna produces a sharp field maximum along the square line between the two strips forming the transmission line. ${ }^{10}$ The field at the source and the image planes was then measured along the center line of the antenna (yellow dashed line in Fig. 4). The field measurement was made following the same procedure as in a previous work: ${ }^{10}$ a probe consisting of a small loop antenna was moved along the dashed line shown in Fig. 4(c) and the received signal was recorded. The probe was moved by steps of length equal to the lens periodicity $a$, and was located at the center of the loops at each step. This measurement was repeated for several values of the lens width $d$ (which was also the distance between the lens and the source antenna). The results of these measurements are shown in Figs. 5(a) and 5(b). The black solid lines in Figs. 5(a) and 5(b) correspond to the measurement of the field that produces the antenna at the source plane. The remaining lines correspond to the measurement of the field at the image plane for several values of $d / a$. The red solid lines in Figs. 5(a) and 5(b) correspond to the measurement of the image field in the UL-MI lens and in the CL-MI lens for $a$. As can be seen, in both cases there is a good agreement between the image field and the source field both qualitatively and quantitatively. The blue solid lines in Figs. 5(a) and 5(b) show that, for $d / a$ $=0.45$, only the UL-MI lens reproduces, at least qualitatively, the source field. Finally, the green solid lines in Figs. 5(a) and 5(b) makes it clear that both lenses fail when the width to periodicity ratio is $d / a=0.6$. When the lenses fail [green line in Fig. 5(a), blue and green lines in Fig. 5(b)], the measurements show a maximum at the center of the antenna (which at the source was a minimum) and an oscillating decay at both sides of this maximum. This behavior is typical, and shows that in both cases the failure of the lens is due to the excitation of MISWs, as was already observed by Sydoruk. $^{11}$

\section{DISCUSSION}

The experiments reported in the previous section clearly show that the performance of MI lenses can be substantially improved by reducing the inter-resonator magnetic coupling, in agreement with the theoretical analysis. Limitations of this strategy observed in the experiments can be attributed to practical limitations of our photoetching technique, as well as to the effect of couplings between non-nearest neighbors, which have not been taken into account in the theoretical model. The fact that the MI lens fails at high values of $d / a$ due to the excitation of MISWs is also in agreement with the theoretical model: when the ratio $d / a$ increases, the two MISW bands overlap (see Fig. 3) and MISWs are excited at any frequency, thus preventing image formation. Regarding lens resolution $\Delta$, if it is defined, as usual, as the minimum distance between two sources that can be resolved by the lens, it is apparent that $\Delta=2 a$ for good MI lenses. This gives a ratio between the lens width and the resolution $d / \Delta$ $=0.225$ for the best studied case [blue line in Fig. 5(a)]. This value is of the same order as the values usually reported in the literature for other metamaterial subdiffraction devices. ${ }^{20}$ This is a remarkable fact since the physical principles and frequencies of operation of such devices are quite different. At this point it may be worth noting the essential difference between the reported MI lenses and other imaging devices based on MI waves ${ }^{21,22}$ or in other mechanisms ${ }^{23}$ which are able just to translate the image from one side of the device to its other side. This property, although remarkable, does not imply the capability of imaging field distributions located at some distance from the device. Actually these devices could be more properly termed as "guiding" systems (magnetic flux guiding system in the case of the devices reported by Wiltshire $^{21}$ and Zhuromskyy ${ }^{22}$ ). Regarding the ratio between the wavelength and the resolution, which is sometimes used as a figure of merit for superlenses, for the best reported MI lens [blue line in Fig. 5(a)] it is $\lambda / \Delta \sim 100$. However, this remarkably high value is not of special physical significance since, as was already pointed out in the Introduction, the real challenge for metamaterial superlenses is to improve the $d / \Delta$ ratio [see Eq. (1)], not the $\lambda / \Delta$ one. In fact, the very high 
$\lambda / \Delta$ ratios that can be achieved by the studied MI lenses are simply due to the very low frequency of operation of such devices.

The ability to image objects smaller than the wavelength is something long established in magnetic resonance imaging (MRI), where imaged objects are very small as compared to the wavelength of the radiofrequency fields used to obtain the image. Therefore, MRI appear as the natural field of application of MI lenses. As is well known, the generation of images in MRI is based on spatial variations in the phase and frequency of the radiofrequency energy absorbed and emitted by the imaged object. These spatial variations are induced by several static magnetic field gradients, and the image is built from signals measured by a receiving coil that do not contain information about the relative location of the emitting magnetic dipoles. The measurement of the signals along time provides the Fourier transform of the distribution of the radiofrequency field magnetic field emitted by the object as a function of spatial frequencies. To obtain the distribution of the radiofrequency fields in the spatial domain, and thus an image of a single slice of tissue, it is necessary to record many repeated measurements (typically 256 or 512) and then to process the set of signals by means of a fast Fourier transform algorithm. Therefore, the generation of images in conventional MRI is not a real-time process. Since real-time imaging is of interest for many medical applications (for instance in cardiac imaging or magnetic resonance angiography), the possibility to obtain the field distribution directly in the spatial domain without signal processing would be of great interest. Since a MI lens directly placed on the skin can "translate" the magnetic field distribution in a slice of tissue located at a distance $d$ inside the human body to another plane located at a distance $d$ outside the skin (see Fig. 1), this would provide direct real-time MRI without any signal processing. However, this first and "naive" idea, although interesting, is of limited application due to the limited performances of present MI lenses. As has been shown all along in the paper, the width to periodicity ratio $d / a$ of any practical MI lens cannot be made very high, even if very precise designs and superconducting rings are used. Since for the aforementioned application this ratio also gives the ratio between the penetration and the resolution, direct MRI by MI lenses would provide resolutions of the same order as the penetration beneath the skin. Of more practical interest, at least at the present stage of the technology, would be a "mixed" technique combining signal processing with direct imaging by MI lenses. During the last years, many efforts have been made in order to reduce acquisition time in conventional MRI. The most successful approach in this direction has been given by the different parallel imaging techniques recently developed. ${ }^{16,24-27}$ Parallel imaging makes use of arrays of resonant receiving coils which can directly provide some extra spatial information about the relative location of the dipoles. The different parallel imaging techniques make use of different algorithms to include this extra spatial information in the signal processing. ${ }^{24-27}$ The main limitations of such techniques come from the fact that the sensitivity of the coils of a single array decays with the distance to the array, and that the individual coils in the array do not have localized sensitivity patterns. However, using a MI lens (which in essence only implies using a pair of coupled arrays of resonant coils instead of using a single array as usual in parallel imaging) can help to overcome such limitations, since using the MI lens is equivalent to having the receiver immersed in the body, at a distance $d$ beneath the skin. Although the penetration/resolution ratio would be always limited by the $d / a$ ratio of the lens, we feel that a significant improvement in MRI acquisition times could be obtained by combining imaging by MI lenses and MRI parallel imaging techniques. More research in this direction is in progress.

\section{CONCLUSION}

MI lenses are perhaps the simplest subdiffraction imaging devices that can be designed to operate in the magnetostatic limit, from radiofrequencies to terahertz. This simplicity comes from its very basic design, which consists of a pair of coupled parallel arrays of magnetic resonators (capacitively loaded rings, SRRs, or other designs). MI lenses share with other previously reported superlenses the ability of imaging with subdiffraction resolution field distributions located at some distance of the lens. In MI lenses, the ratio between the imaging distance and the resolution is of the same order as the ratio between the lens width and periodicity. It has been theoretically and experimentally shown that this ratio is mainly limited by the coupling between nearest resonators, and by the resonator's losses. By partially overlapping the resonators in the arrays, this coupling can be significantly reduced, and the MI lens performance can be substantially improved. Interesting practical applications of MI lenses in magnetic resonance imaging can be foreseen.

\section{ACKNOWLEDGMENTS}

This work has been partially supported by the Spanish Ministry of Science and Education under Contract No. TEC2004-04249-C02-02 and by Junta de Andalucia under Contract No. P06-TIC-01368. The authors also thank Professor Laszlo Solymar for helpful suggestions during the realization of this work.

${ }^{1}$ J. B. Pendry, Phys. Rev. Lett. 85, 3966 (2000).

${ }^{2}$ R. Marques and J. Baena, Microw. Opt. Technol. Lett. 41, 290 (2004).

${ }^{3}$ M. C. K. Wiltshire, J. B. Pendry, and J. V. Hajnal, J. Phys.: Condens. Matter 18, L315 (2006).

${ }^{4}$ N. Fang, H. Lee, C. Sun, and X. Zhang, Science 308, 534 (2005).

${ }^{5}$ R. Marques, F. Mesa, and F. Medina, Appl. Phys. Lett. 86, 023505 (2005).

${ }^{6}$ S. Maslovski, S. Tretyakov, and P. Alitalo, J. Appl. Phys. 96, 1293 (2004).

${ }^{7}$ M. J. Freire and R. Marques, Appl. Phys. Lett. 86, 182505 (2005).

${ }^{8}$ K. Guven and E. Ozbay, Opto-Electron. Rev. 14, 213 (2006).

${ }^{9}$ E. Shamonina, V. A. Kalinin, K. H. Ringhofer, and L. Solymar, J. Appl. Phys. 92, 6252 (2002).

${ }^{10}$ M. J. Freire and R. Marques, J. Appl. Phys. 100, 063105 (2006)

${ }^{11}$ O. Sydoruk, M. Shamonin, A. Radkovskaya, O. Zhuromskyy, E. Shamonina, R. Trautner, C. J. Stevens, G. Faulkner, D. J. Edwards, and L. Solymar, J. Appl. Phys. 101, 073903 (2007).

${ }^{12}$ V. A. Podolskiy and N. A. Kuhta, Appl. Phys. Lett. 87, 231113 (2005).

${ }^{13}$ It may be worth noting that these poles appear for imaginary values of $k_{z}$. Therefore, the incident wave is evanescent and the modulus of the transmission coefficient is not limited by energy conservation.

${ }^{14}$ R. E. Collin, Field Theory of Guided Waves, 2nd ed. (IEEE Press, New York, 1990).

${ }^{15}$ L. Solymar (private communication).

${ }^{16}$ C. J. Hardy, H. E. Cline, R. O. Giaquinto, T. Niendorf, A. K. Grant, and D. 
K. Sodickson, Magn. Reson. Med. 55, 1142 (2006).

${ }^{17}$ R. D. Black, P. B. Roemer, and O. M. Mueller, IEEE Trans. Biomed. Eng. 41, 195 (1994)

${ }^{18}$ S. E. Hurlston, W. W. Brey, S. A. Suddarth, and G. A. Johnson, Magn. Reson. Med. 41, 1032 (1999).

${ }^{19}$ M. J. Lancaster, T. S. M. Maclean, Z. Wu, A. Porch, P. Woodall, and N.NcN. Alford, IEE Proc., Part H: Microwaves, Antennas Propag. 139, 149 (1992).

${ }^{20}$ For instance, the silver lens reported by Fang (Ref. 4) has a width of 35 $\mathrm{nm}$, and is able to resolve a periodic pattern of line sources, with a periodicity of $120 \mathrm{~nm}$. Therefore, for this device it is $d / \Delta=0.291$, a value quite similar to the reported value for the best UL-MI lens.
${ }^{21}$ M. C. K. Wiltshire, J. B. Pendry, I. R. Young, D. J. Larkman, D. J. Gilderdale, and J. V. Hajnal, Science 291, 849 (2001).

${ }^{22}$ O. Zhuromskyy, E. Shamonina, and L. Solymar, Opt. Express 13, 9299 (2005).

${ }^{23}$ P. A. Belov, Y. Hao, and S. Sudhakaran, Phys. Rev. B 73, 033108 (2006).

${ }^{24}$ D. K. Sodickson and W. J. Manning, Magn. Reson. Med. 38, 591 (1997).

${ }^{25}$ K. P. Pruessmann, M. Weiger, M. B. Scheidegger, and P. Boesiger, Magn. Reson. Med. 42, 952 (1999).

${ }^{26}$ M. A. Griswold, P. M. Jakob, M. Nittka, J. W. Goldfarb, and A. Haase, Magn. Reson. Med. 44, 602 (2000).

${ }^{27}$ M. P. McDougall and S. M. Wright, Magn. Reson. Med. 54, 386 (2005). 